Andreas Trotzke* and Anna Czypionka

\title{
Non-canonical questions from a comparative perspective: Introduction to the special collection
}

https://doi.org/10.1515/lingvan-2020-0106

Received October 27, 2020; accepted October 28, 2020

Abstract: In this brief introduction, we provide an overview of this special collection on a variety of linguistic means to convey non-canonical interpretations of the speech act of questioning.

Keywords: comparative linguistics; interrogatives; non-canonical questions

\section{Introduction}

The aim of this special collection is to bring together work on non-canonical questions from a comparative perspective. By 'non-canonical' questions we mean questions that do not request information from the addressee, but rather tell us something about the speaker's epistemic and/or emotional state (e.g., echo questions, rhetorical questions, and surprise questions). While there has been a lot of cross-linguistic research on the speech act of questioning over the last 50 years or so (see Dayal 2016 for a comprehensive overview), our knowledge about cross-linguistic patterns of non-canonical questions has seen a rapid development only recently - and research in this domain is conducted within all core areas of linguistics: semantics/pragmatics (e.g., Beltrama et al. 2020), phonetics/phonology (e.g., Braun et al. 2019; Dehé and Braun 2020), and syntax (e.g., Giorgi and Dal Farra 2019).

The contributions in the present volume explore how semantic/pragmatic, prosodic, and syntactic factors affect the meaning of interrogative sentences in such a way that those factors yield a non-canonical reading. The individual papers thereby demonstrate for different languages how for instance modal components such as speaker attitude, but also general discourse requirements (particularly information structure) interact with the speech act of questioning.

Before we sketch the individual chapters of the volume, we would like to thank all the scholars who reviewed the contributions to this volume and who provided extremely helpful feedback. Thanks also to Eva Wittenberg, our Area Editor, and to the Linguistics Vanguard editorial team for their support during the editing and production process. Last not least, we would also like to thank the German Research Foundation (DFG research unit “Questions at the Interfaces," FOR 2111) and the German Academic Exchange Service/DAAD (PPP project Surprise Questions from a Comparative Perspective; grant no. 57444809) for financial support. Andreas Trotzke additionally acknowledges financial support from the EU Horizon 2020 COFUND scheme for the project "Functional categories and expressive meaning" (grant no. 2017-BP00031).

\section{The contributions}

The first four papers of this collection deal with the phenomenon of non-canonical questions from the perspective of semantics and pragmatics. In particular, the paper by Lucia M. Tovena ("A comparative corpus

*Corresponding author: Andreas Trotzke, University of Konstanz, Konstanz, Germany, E-mail: andreas.trotzke@uni-konstanz.de Anna Czypionka, University of Konstanz, Konstanz, Germany, E-mail: anna.czypionka@uni-konstanz.de 
study on a case of non-canonical question") focuses on French wh-interrogatives with the element comment 'how' in their reason interpretation. The author first highlights that the non-canonicity of those questions is due to the modified semantic type of the domain of the $w h$-item, and then she continues by exploring potential constraints of this question type by means of corpus data. Specifically, Tovena demonstrates that commentquestions in French can be used to invite or propose answers with respect to the situation described by the prejacent, which distinguishes them from exclamatives. Based on her corpus data, the author also points out that this type of non-canonical question is not only restricted to informal language. The second paper on the semantics and pragmatics of non-canonical questions deals with "Interpreting high negation in Negative Interrogatives: The role of the Other.” The authors Pierre Larrivée and Alda Mari deal with English questions such as Don't you speak Italian? and paraphrase their reading as a bias on the part of the speaker towards the underlying positive proposition. Given their new observation that Negative Interrogatives also involve uncertainty with respect to the relevant proposition (reminiscent of epistemic modals), the authors provide a speech-act theoretic analysis and claim that the non-canonical interpretation of such questions can be derived by two operators which they call 'Speaker' and 'Other', and which represent different sources of information. By proposing those operators, Larrivée and Mari postulate a direct mapping from syntax to semantics and pragmatics that can explain the morphosyntactic and interpretative properties of Negative Interrogatives.

Lisa Brunetti, Lucia M. Tovena, and Hiyon Yoo ("French questions alternating between a reason and a manner interpretation") investigate the interpretation of French comment ('how') questions that - depending on context - can be interpreted as asking for the manner or the reason of the denoted event. The results of their rating study show that reason-comment is interpreted as expressing surprise in addition to a request for information. This suggests that surprise questions are indeed truly interrogative (unlike rhetorical questions), but different from plain information-seeking questions, and that expectation disconfirmation is wired in the semantics of reason-comment. The authors offer an insightful link to an earlier production study, showing that the current study essentially mirrors prosodic findings, particularly a higher inter-speaker variability for the interpretation and production of reason-comment when compared to manner-comment. Brunetti et al. propose that this higher variability mirrors the more complex semantics of reason- compared to manner-comment questions.

The fourth and last contribution on the semantics and pragmatics of non-canonical questions is by Andreas Trotzke and Anna Czypionka. In their paper on "The pragmatics of surprise-disapproval questions: An empirical study," they investigate a type of non-canonical question that expresses the speaker's surprise and/or disapproval with respect to the event denoted by the propositional part of the question. Their paper empirically assesses theoretical claims about the illocutionary status of surprise-disapproval questions by exploring their pragmatics in an acceptability study. The authors highlight that the results of their experiment are particularly compatible with a broad theory that assumes that pragmatic phenomena like surprisedisapproval questions are truly 'mixed' (speech-act and clause) types in the sense that we do not have to postulate a distinct illocutionary status (or, in syntactic terms, a distinct projection) in order to explain them theoretically. In this regard, Trotzke and Czypionka's account dovetails nicely with both Brunetti et al.'s findings and Celle and Pélissier's study (see below) on the interrogative nature of surprise questions.

The next two articles in our special collection capitalize on the morpho-syntactic properties of noncanonical questions. Yoshio Endo's contribution on "Non-standard questions in English, German, and Japanese” deals with syntactic properties of so-called discourse (aka 'modal') particles, particularly in Japanese, and on this basis proposes that non-canonical questions can be classified into two types: the speaker-oriented type and the addressee-oriented type. Endo goes on to discuss the properties of Japanese sentence-final particles, showing that the addressee-oriented type of non-canonical questions can be further divided into the counter-expectation type and the aggressive-attitude type. The author provides a syntactic analysis of all relevant particles and readings, and he compares his observations on Japanese to similar readings and functional linguistic categories in German and English. The second paper on the syntactic representation of non-canonical readings of questions deals with particle-like elements as well.

Johannes M. Heim and Martina E. Wiltschko's paper "Timing of belief as a key to cross-linguistic variation in common ground management" offers a cross-linguistic comparison of the sensitivity to Timing-of- 
Belief for tag questions in English, Spanish, and German. The results of their study match their assumption that confirmational particles in different languages come with different and graded preferences regarding Timing-of-Belief. According to the authors, their findings showcase the need to base models of common ground management on a variety of languages. They sketch a detailed syntactic proposal for related phenomena as well as promising directions for future work, including a possible relation between a language's sensitivity to Timing-of-Belief and the morphosyntactic property of tense.

The last two articles of this collection deal with the phonology of non-canonical questions. In their contribution "The prosody of French rhetorical questions," Claire Beyssade and Elisabeth Delais-Roussarie present a production study comparing string-identical rhetorical and information-seeking questions in French, using a well-established paradigm that allows for future cross-linguistic comparisons. Their findings offer a characterization of the prosodic cues distinguishing both readings, showing that a whole set of prosodic cues, rather than a single prosodic or intonational pattern, are important for interpretation. The authors propose that the divide between rhetorical and information-seeking questions is not categorical, and that the interplay of multiple prosodic cues possibly reflects different semantic and pragmatic features involved.

The last contribution to this special collection by Agnès Celle \& Maud Pélissier on "Surprise questions in spoken French" characterizes the prosody of two types of non-canonical wh-questions in French (C'est quoi, ce $N$, 'What is this N?', and Qu'est-ce que ... , 'What is/Why is ... '). According to the authors, those question types signal surprise. In a production study, they compare both constructions to string-identical canonical questions. Their results show that prosodic patterns for both constructions deviate from the canonical realizations in the same dimensions at the level of the sentence and question words. While these deviations share some properties with the prosodic patterns found earlier for rhetorical questions, the current study also shows that surprise questions differ prosodically from both rhetorical and information-seeking questions, strengthening the claim that this type of question cannot simply be reduced to one or the other.

\section{References}

Beltrama, Andrea, Erlinde Meertens \& Maribel Romero. 2020. Alternative questions: Distinguishing between negated and complementary disjuncts. Semantics and Pragmatics 13. 5.

Braun, Bettina, Nicole Dehé, Jana Neitsch, Daniela Wochner \& Katharina Zahner. 2019. The prosody of rhetorical and informationseeking questions in German. Language and Speech 62. 779-807.

Dayal, Veneeta. 2016. Questions. Oxford: Oxford University Press.

Dehé, Nicole \& Bettina Braun. 2020. The intonation of information seeking and rhetorical questions in Icelandic. Journal of Germanic Linguistics 32. 1-42.

Giorgi, Alessandra \& Chiara Dal Farra. 2019. On the syntax/pragmatics interface: Expressing surprise and disapproval. Intercultural Pragmatics 16. 335-361. 